\title{
Analysis of the repeatability of fuel spray indexes in a spray-guided direct-injection spark-ignition engine
}

\begin{abstract}
The development and research works on liquid fuel injection in spark-ignited direct injection (SIDI) engines, apart from so common in recent years simulation methods, still have a significant cognitive substrate. This is related to experimental research on repeatability of combustion process using multi- and mono-cylinder test engines and Rapid Compression Machines. The repeatability of preparation and delivery processes has immediate impact on repeatability of combustion process. Except for the necessity of obtaining the repeatability of fuel amounts, the repeatability of injected fuel spray is required. The penetration range and spray area in combustion chamber have direct impact on mixture creation and formation. The optical research on fuel injection has been made in order to determinate its repeatability. The research on unrepeatability of fuel spray propagation has been conducted using piezoelectric injectors of outward-opening type, being primary elements of the spray-guided combustion systems. The results of research were presented in the form of index of variation of the selected parameters. The evaluation of the results of the optical research concerns radial spray penetration and fuel spray velocity. Unrepeatability has been presented with coefficient of variation of radial penetration in relation to the time of injection duration. It has been observed that the coefficients of various parameters are lower with longer times of fuel injection.
\end{abstract}

Key words: spray atomization, optical research, image analysis, fuel penetration

\section{Introduction}

Combustion systems of SI (spark ignition) engines with direct injection at the time of mixture ignition require very precise synchronization of time of fuel spray penetration in the direction of the spark plug. For combustion systems with fuel amount split into several parts such requirements are even more stringent. Ignition dose should be delivered at a specified time before spark plug discharge, as in its immediate surrounding is required a mixture enabling a reliable ignition. Research carried out by Waltner et al. [17] emphasizes the importance of this issue, especially in the aspect of a fuel injection control system and mixture formation in the combustion chamber.

The research by Waltner et al. [17] proves that the range of correct ignitability of the mixture with a three-part injection applied is much wider than that for the two-part injection. It is so due to a better distribution of the mass of fuel injected during injection of a dose split into three parts compared to that split into two parts. It can be therefore concluded, that an increase of the fuel amount (an increase of the engine load) forces the use of a multi-part fuel injection. Multi-part injection enables a better control of the quality of the composition of a mixture formed around the spark plug. The central location of the injector close to the spark plug makes it possible to obtain the value of the combustion air factor $\lambda$ guarantying appropriate ignitability of the mixture.

For the high-speed flow of fuel from the injector (of about $100 \mathrm{~m} / \mathrm{s}$ ) a proper fuel atomization occurs. In the piezoelectric injectors (direct injection) of gasoline atomized under pressure higher than $5 \mathrm{MPa}$ (in the tests the pressure of $20 \mathrm{MPa}$ was applied) the loss of spray stability does not involve the formation of a membrane. Due to the waves of short lengths it has not enough time to break up into rings [2]. This means an increase of the Weber number and al- lows determination of the impact of external factors on the drops formation. The secondary breakup of drops ensues from aerodynamic force in the area of increased dynamic pressure of the gas when the aerodynamic force is greater than the forces of surface tension. The greater the Weber number is, the smaller the dimensions of drops after the secondary break-up are [2]. Secondary breakup of drops is a very complex and insufficiently studied phenomenon; for this reason, the values of Weber number are specified in quite a wide range.

In paper [13], it was found that in the spark ignition engines with direct injection (utilizing multi-orifice injector), Weber number ranges from 1 to 250 , however for the largest number of drops this value ranges from 1 to 30 .

Secondary breakup of drops is divided into number intervals [4]. When the drops of $\mathrm{D}$ diameter are characterized by a low Weber number $(\mathrm{We}<12)$, most often their deformation, and not their breakup, takes place. Taking into consideration the liquid viscosity during breakup causes the increase of the value of Weber number required for breakup. Liquid viscosity $\mu_{\mathrm{c}}$ is related to its density $\rho_{\mathrm{c}}$ and surface stresses $\sigma_{c}$ with the use of Ohnesorge number [4]:

$$
\mathrm{Oh}=\mu_{\mathrm{c}} /\left(\rho_{\mathrm{c}} \mathrm{D} \sigma_{\mathrm{c}}\right)^{0.5}
$$

Division of the secondary atomization into five areas considering the Weber number and Ohnesorge number ranges for gasoline and diesel are given in the papers $[4,5]$. These papers indicate a relationship between the type of the secondary breakdown and the Weber number. It was shown that the Ohnesorge number has an impact on the breakdown of drops only if its value is greater than 1 (Fig. 1).

Thus, obtaining proper fuel atomization is closely related to the uniformity and repeatability of its atomization. Any change to atomization leads to different spray penetration and 
this generates different fuel speed. This leads to a variable breakup and non-uniform formation of drops.

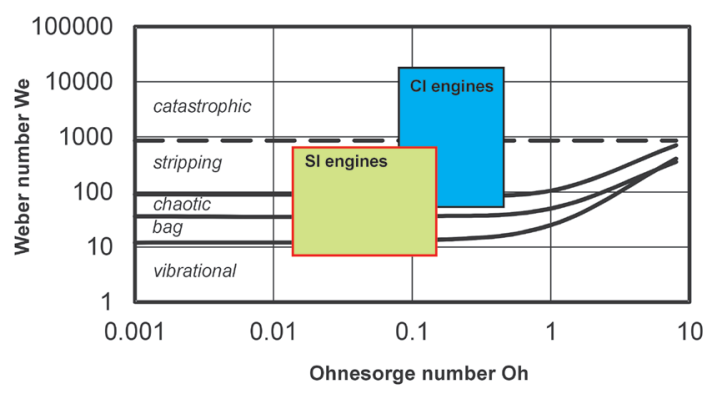

Fig. 1. Map of secondary atomization regimes as functions of Ohnesorge and Weber numbers $[4,5]$

Previous research on non-uniformity of fuel injection from injectors of outward-opening type involves mainly issues of the linear analysis of fuel spray penetration. Paper by Peterson [11] concerning the ignitability of the mixture formed in the spray-guided systems (SG) focuses on linear distribution of the spray speed and its ignitability in the surrounding of the spark plug. Oh and Bae [10] conducting tests with the use of a constant-volume chamber determined only the linear penetration of the fuel spray. Studies of the radial propagation of the fuel spray were conducted by El-Asrag et al. [6]; however, they involved the issue of simulation of the flow of fuel from the injector in a micro-scale. The focus was on radiational issues caused by the dynamics of the fuel spray flow.

\section{The reasons for undertaking the subject}

The above-presented aspects concerning the ignitability of the mixture are closely related to the quality of its formation. Obtaining an effective ignitability of the mixture (with high probability of ignition) requires a uniform distribution of the injected fuel spray and also its proper breakup. Nonuniformity might be mainly caused by variations in the regulations of the injector (for new injectors) or non-uniform wear (exploited injectors).

Due to the expected non-uniformity in fuel spray penetration the analyses were undertaken to identify the subsequent fuel spray penetrations on the basis of optical tests. Such studies are possible during the simulation analysis; however, the conducted experiments show some imperfections of the optical tests.

The studies presented below were designed to obtain answers to the question of uniformity of the fuel spray penetration during high-pressure injection of fuel from the system of outward-opening type. This uniformity was determined on the basis of:

a) the radial penetration of the fuel spray $P$ obtained for the injector; these tests were carried out for the round angle of fuel spray atomization with resolution of 2 degrees; as a criterion for the maximum permissible value of nonuniformity was assumed the $5 \%$ coefficient of variation of the radial penetration in relation to the circular profile of such a flow. b) radial penetration averaged from the measuring cycles; these studies were carried out in range up to $1 \mathrm{~ms}$; the criterion of maximum permissible non-uniformity was also established at $5 \%$ variation from the mean of such radial penetration;

c) radial speed (V) of spray of injected fuel; this parameter was specified indirectly using the penetration calculated previously for subsequently recorded images and analyzing the time of subsequent records; the criterion of maximum permissible non-uniformity was also established at $5 \%$ of coefficient of variation $-\mathrm{CoV}(\mathrm{V})$.

\section{The research object and apparatus}

The study was carried out using a piezoelectric injector with outward opening of the needle. The new Siemens injector was used to eliminate the influence of operating conditions (wear) on the assessment of uniformity of fuel atomization. In the tests was used an injector with static fuel flow of $35 \mathrm{~g} / \mathrm{s}$ at $10 \mathrm{MPa}$, and the value of the maximum fuel pressure of up to $30 \mathrm{MPa}$. The angle of the spray atomization was 90 degrees and the minimum dose was $2 \mathrm{mg} / \mathrm{inj}$. The needle stroke was ranging in different sources from $30 \mu \mathrm{m}$ [18] to $36 \mu \mathrm{m}$ [9]. The injector was controlled by a voltage of $190 \mathrm{~V}$ and minimum time of its opening was about $0.15 \mathrm{~ms}$ [1]. In the tests was used petrol fuel with a density of 720$775 \mathrm{~kg} / \mathrm{m}^{3}$ (at temperature of $15^{\circ} \mathrm{C}$ ) with a sulphur content of up to $10 \mathrm{ppm}$ and the octane number of 95 .

Optical tests of fuel atomization and determination of geometric indexes of the fuel spray were conducted with the injector mounted in the constant-volume chamber with fuel at a pressure of $20 \mathrm{MPa}$ (measured by AVL sensor SL31D2000 with a resolution of $0.001 \mathrm{MPa}$ and accuracy of $1 \% \mathrm{FS}$ - full scale). The back-pressure of the air in the test chamber was $2 \mathrm{MPa}$ (as measured by the Keller PA21 SR sensor with a resolution of $0.001 \mathrm{MPa}$ and reading accuracy of $2 \% \mathrm{FS}$ ). The injection duration was established at 0.3 and $0.7 \mathrm{~ms}$ (controlled by an external system - a sequencer). The tests were carried out with 20 repetitions of fuel spray atomization at each injection.

The test chamber was lit with two $500 \mathrm{~W}$ halogen lamps each, placed in opposition in order to obtain the maximum value fuel spray luminance. The diameter of the quartz

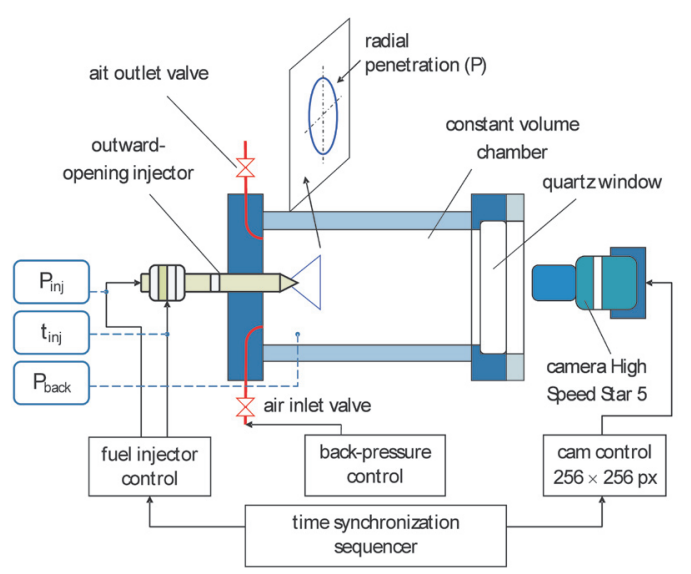

Fig. 2. The test bench for testing the repeatability of fuel injection 
window enabling optical access was $90 \mathrm{~mm}$. The view of the testing bench is presented in Fig. 2.

The optical observations were conducted using the High Speed Star 5 camera by LaVision with CMOS converter. The camera was equipped with a Nikon Nikkor lens, $50 \mathrm{~mm}$ 1:1.4 (D) with the shutter set to 8. Images were recorded with a frequency of $20 \mathrm{kHz}$ at a resolution of $256 \times 256$ pixels. The characteristics of the electrical signal ( $5 \mathrm{~V}, \mathrm{TTL})$ for controlling the forced start of fuel injection, the time of its duration as well as the start time of images recording was provided by the sequencer with time resolution of $2 \mathrm{~ns}$ [12].

\section{Optical tests of repeatability of fuel atomization}

\subsection{Optical tests of fuel atomization}

The flat exposition of the recorded images was analyzed. As might be observed in Fig. 2, the radial propagation of injected fuel spray was recorded, which means the camera was parallel to the axis of the injector. Such parallel placement of the injector and the camera enables to get most information on non-uniformity of radial propagation of fuel spray. Exemplary results of the tests of atomization at injection pressure of $20 \mathrm{MPa}$, the back-pressure in the test chamber of $2 \mathrm{MPa}$ and two different injection durations are shown in Fig. 3.
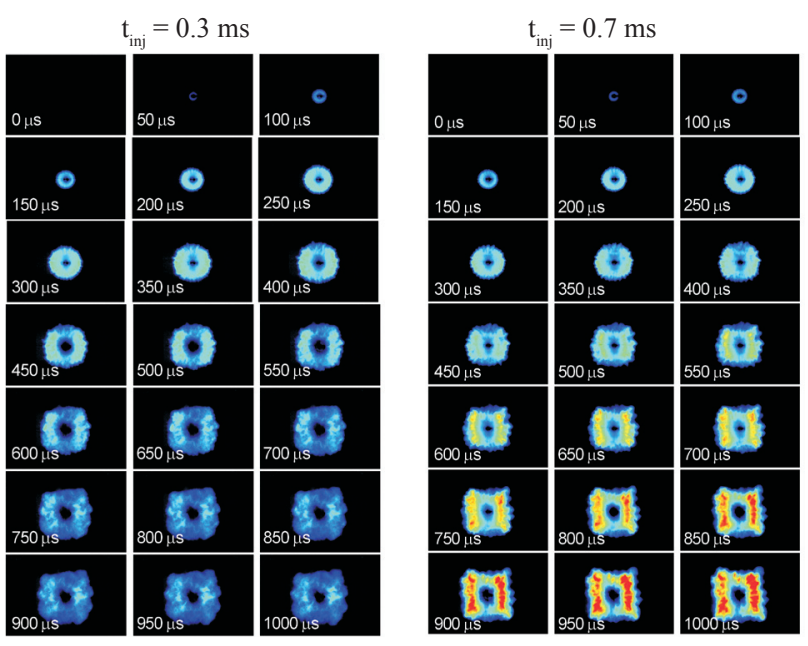

Fig. 3. The recorded images (sample) of fuel atomization at a pressure of injected fuel $\mathrm{P}_{\text {inj }}=20 \mathrm{MPa}, \mathrm{P}_{\text {air }}=2 \mathrm{MPa}$ and the injection duration: a) $0.3 \mathrm{~ms}$, b) $0.7 \mathrm{~ms}$

The change in the intensity of the light indicates a change in the concentration of fuel in the spray. Distinctive, close to square, shape of the fuel spray is the result of the injector design presented, among others, in publications [3, 7].

The paper [7] in a numerical way specifies speed of fuel flow from the injector with the outward opening of the injector needle. The calculations confirmed not only the influence of the shape of the injector hole, but also the influence of the geometry of the needle on the flow of liquid spray. Similarly to the current tests, the right sections of the injected fuel spray close to square were obtained (radial range). This shape is clearly seen for longer injection durations and for long time of fuel atomization recording.

\subsection{Evaluation of specific indexes of fuel atomization}

The photos presented above are the basis for the analysis of the radial penetration of fuel spray in relation to particular value of the round angle. The analysis was conducted for angle increments of $\Delta \alpha=2^{\circ}$ for the analyzed subsequent penetrations of fuel spray with time interval of $0.05 \mathrm{~ms}$. An example of such penetration for two different injection durations is shown in Fig. 4. This figure shows 20 consecutive radial penetrations of fuel spray from $0.05 \mathrm{~ms}$ to $1 \mathrm{~ms}$.

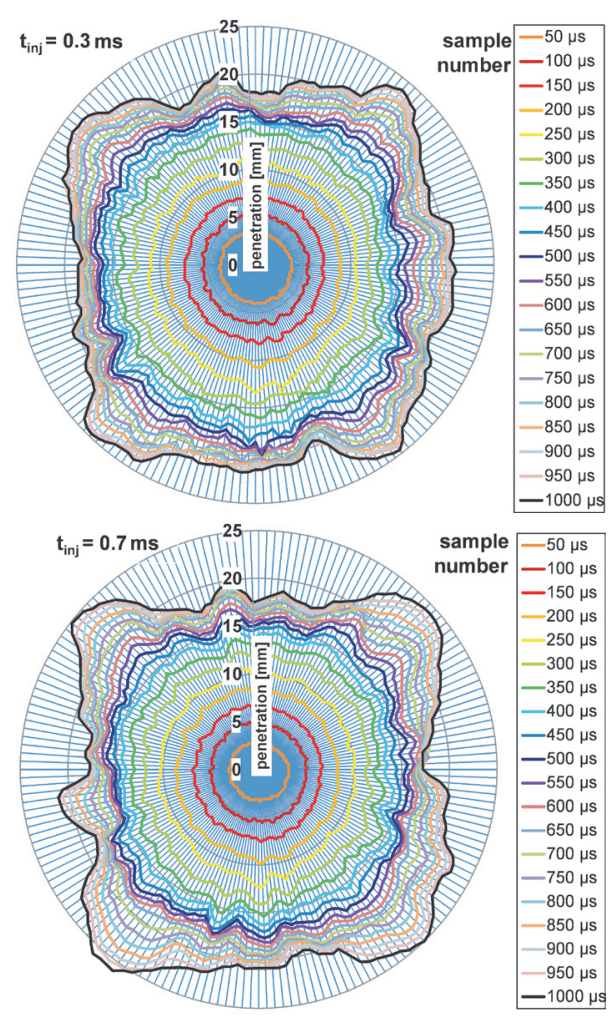

Fig. 4. Analysis of the fuel penetration for two different injection durations with the filming speed of $20 \mathrm{kHz}$

Significant deviations from the mean value are observed for the atomization time of $\mathrm{t}=0.35 \mathrm{~ms}$ and for further atomization of the fuel spray. These deviations are more significant for longer time of fuel injection. However, the change in shape of the spray from round to square takes place at the same time of atomization $\mathrm{t}=0.6 \mathrm{~ms}$.

A detailed analysis of the index of fuel penetration nonuniformity indicates the presence of high value of $\mathrm{CoV}(\mathrm{P})$ at minimum atomization time $(\mathrm{t}=0.05 \mathrm{~ms})-$ Fig. 5a. For the injection duration of $\mathrm{t}=0.3 \mathrm{~ms}$ the $\mathrm{CoV}(\mathrm{P})$ value ranged from $7 \%$ to $10 \%$. During atomization in the time ranging from 0.1 to $0.2 \mathrm{~ms}$ the value of deviations is reduced to approximately $5 \%$. Later the non-uniformity of the radial spray penetration increases, which is indicated by increasing value of the $\mathrm{CoV}$ factor. It obtains the maximum value of approximately $10 \%$ for atomization time of $t=1 \mathrm{~ms}$. The analysis of this non-uniformity for the injection duration $\mathrm{t}_{\text {inj }}=0.7 \mathrm{~ms}$ indicates smaller radial deviation of the fuel spray penetration - Fig. $5 \mathrm{~b}$. Smaller values of these disper- 
sions are observed only for the initial and middle phases of the atomization time. The $\operatorname{CoV}(\mathrm{P})$ obtains values below $5 \%$ for the atomization time ranging from $\mathrm{t}=0.1 \mathrm{~ms}$ to $0.5 \mathrm{~ms}$ for the selected characteristics. However, it should be noted that for all analyzed fuel atomization processes for the time ranging from 0.1 to $0.4 \mathrm{~ms}$ all repetitions have met the limit of $5 \%$ non-uniformity of the $\mathrm{CoV}$ index.

It follows from the above that, during the injection of small amounts $\left(\mathrm{t}_{\text {inj }}=0.3 \mathrm{~ms}\right)$ the penetration of the fuel spray is uniform only up to $0.2 \mathrm{~ms}(\mathrm{CoV}(\mathrm{P})<5 \%)$ (Fig. 5), while for large injected amounts $\left(\mathrm{t}_{\mathrm{inj}}=0.7 \mathrm{~ms}\right)$ the radial penetration is uniform even up to $0.5 \mathrm{~ms}(\mathrm{CoV}(\mathrm{P})<5 \%)$. This means that for the injector of outward-opening type the ignition of small amounts at short intervals is required, and the intervals can be extended (almost twice) during injection of large amounts. The presented injection duration $(\mathrm{t}=0.3$ $\mathrm{ms}$ and $\mathrm{t}=0.7 \mathrm{~ms}$ ) ensure the uniformity of distribution of the fuel spray penetration, and thus can increase the probability of ignition as the variations in the radial penetrations are insignificant. a)

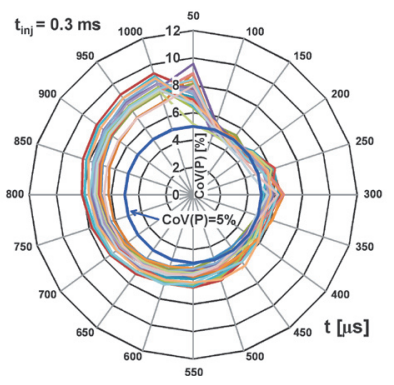

b)

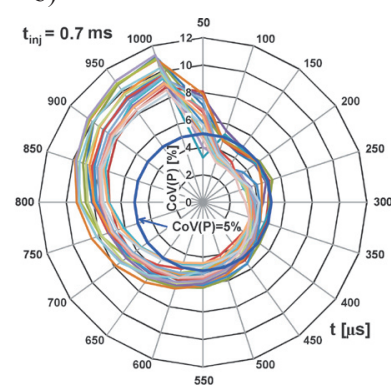

Fig. 5. Specific coefficients of variation of the radial penetration related to the subsequent times of fuel atomization: a) a small fuel amount of $\mathrm{t}_{\text {inj }}=0.3 \mathrm{~ms} ; \mathrm{b}$ ) a large fuel amount $\mathrm{t}_{\text {inj }}=0.7 \mathrm{~ms}$

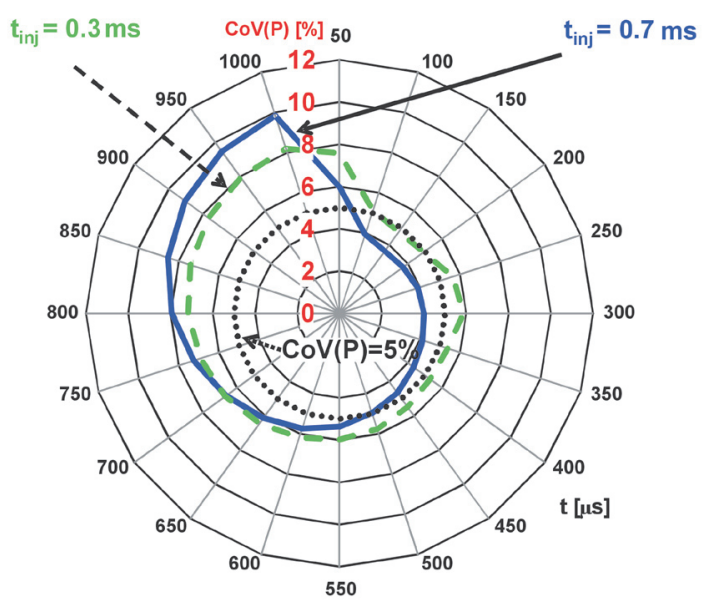

Fig. 6. The average values of the non-uniformity of the radial distribution on circular profile of the fuel spray for different amounts of fuel injected

Later in the article the average values of the geometric indexes of injected fuel spray are analyzed: the average radial penetration of fuel spray and the average value of speed of the fuel spray front.

\section{The evaluation of average values of indexes of fuel atomization}

\subsection{Evaluation of the uniformity of the average radial fuel penetration}

Evaluation of uniformity of the average radial fuel spray penetration was conducted for 20 subsequent repetitions of fuel injections. The analysis was carried out for the time range from $0.05 \mathrm{~ms}$ to $0.3 \mathrm{~ms}$ for the same fuel amounts as previously. The values of the radial penetration of fuel spray are shown in Fig. 7. The most significant changes in the radial penetration of fuel spray were observed during the start of injection. With the time of injection, the reduction in the dispersion of this index was observed.
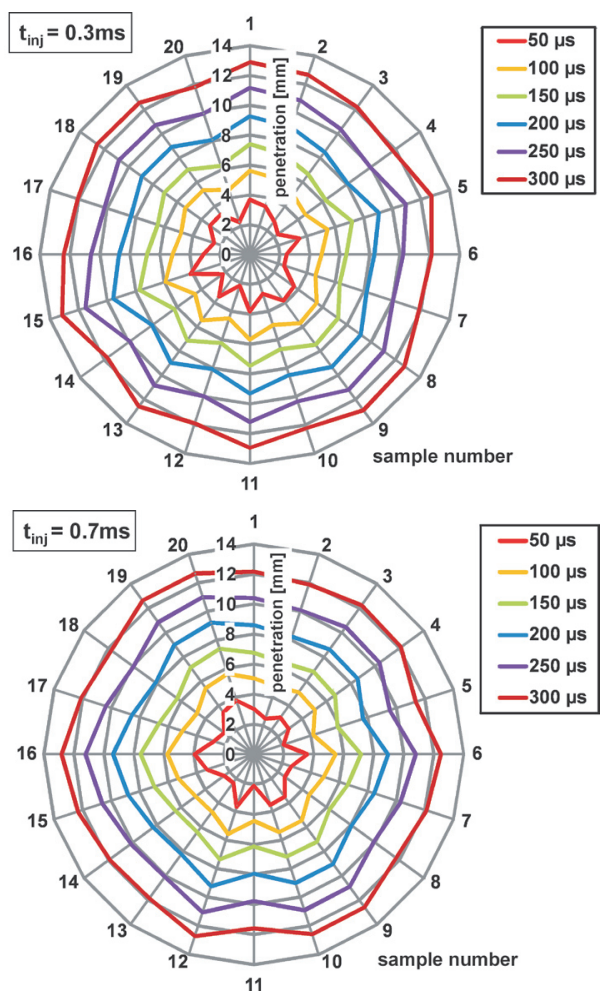

Fig. 7. The results of the radial penetration test of 20 consecutive amounts of injected fuel in the time range from 0.05 to $0.3 \mathrm{~ms}$ (for two amounts with injection durations $\mathrm{t}=0.3 \mathrm{~ms}$ and $\mathrm{t}=0.7 \mathrm{~ms}$ )

The analysis of the results presented in Fig. 7 indicates the existence of significant values of non-uniformity in the radial penetration amounting to $20 \%$ during injection initiation (regardless of the amount). The full analysis of the nonuniformity of the radial penetration of fuel amount indicates the existence of a critical time, after which the values of the non-uniformity of the radial penetration are smaller than 5\%. This critical time falls at approximately $0.2 \mathrm{~ms}$ (Fig. 8). After this time, the dispersions of the values are below $5 \%$, which is acceptable. It should be noted that the obtained time of $\mathrm{t}=0.2 \mathrm{~ms}$, after which the non-uniformity of the penetration reaches the value below 5\% does not depend on the injection duration or on the fuel amount.

The optical analysis of the geometric indexes of fuel spray is possible when on the basis of the subsequent images, the fuel spray penetration might be determined unequivo- 
cally. This penetration depends on a number of factors the most important of which are the fuel pressure and the density of the medium into which the fuel is injected. This density is closely related to the back-pressure of this medium. In the tests presented, the fuel pressure to medium back-pressure ratio was 10:1. For such values the analysis of unequivocally determined fuel spray penetration was conducted. Such tests are possible if the spray penetrates into the analyzed space without increasing the vortex of the front of the fuel spray. The conducted analysis shows that for smaller amounts $(\mathrm{t}=$ $0.2 \mathrm{~ms}$ ) the fuel spray penetrates into the chamber for up to about $0.4 \mathrm{~ms}$ (Fig. 9). After this time there is an increased intensity of radial vortex of the fuel spray which makes it impossible to unequivocally determine the increase of its penetration. Larger values of injection durations (higher amounts) increase this time up to $0.5 \mathrm{~ms}$.
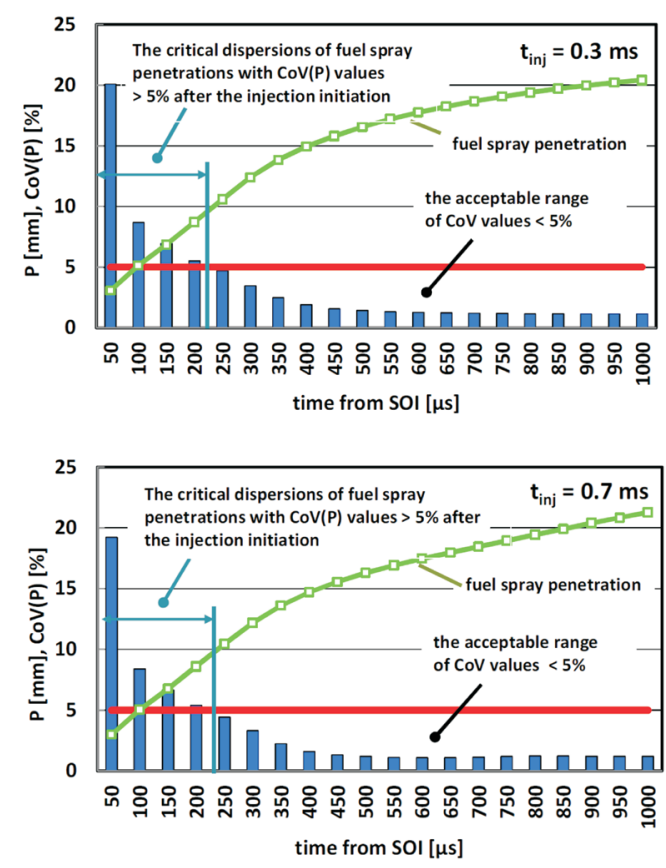

Fig. 8. The analysis of the non-uniformity of the average radial penetration of fuel injected at different injection durations

A confirmation of that analysis is, among others, the paper [15], in which the Authors utilizing the Mie method determined the directions of movement of the atomized fuel. In the initial phase of the injection the air is "displaced" by the atomized fuel. Due to a high relative density of the air, under the influence of resisting forces acting on the atomized fuel a vortex is created which directs the fuel spray outwards. As the spray has the shape of a hollow cone, the air is carried up, which was also shown in the papers $[14,16]$. Then, in conjunction with air resistance forces, another rotating whirl is created (of vortex type). These whirls, depending on the back-pressure, effectively reduce the penetration of the spray.

Complementary to these studies can be studies by Kim et al. [8], in which the velocity of mixture vortex during fuel spray injection was determined. The tests were carried out at the pressure of injection into the constant-volume chamber of $20 \mathrm{MPa}$. From the distribution of the velocity field it ensues that the spray velocity is not greater than $50 \mathrm{~m} / \mathrm{s}$ (after the injection duration $\mathrm{t}=0.5 \mathrm{~ms}$ ). The recirculation zone is formed at the bottom part of the spray, where the vortex of the mixture is dominant. The air flowing out from the inside of an empty cone carries the charge up and outward into the direction of the injector.
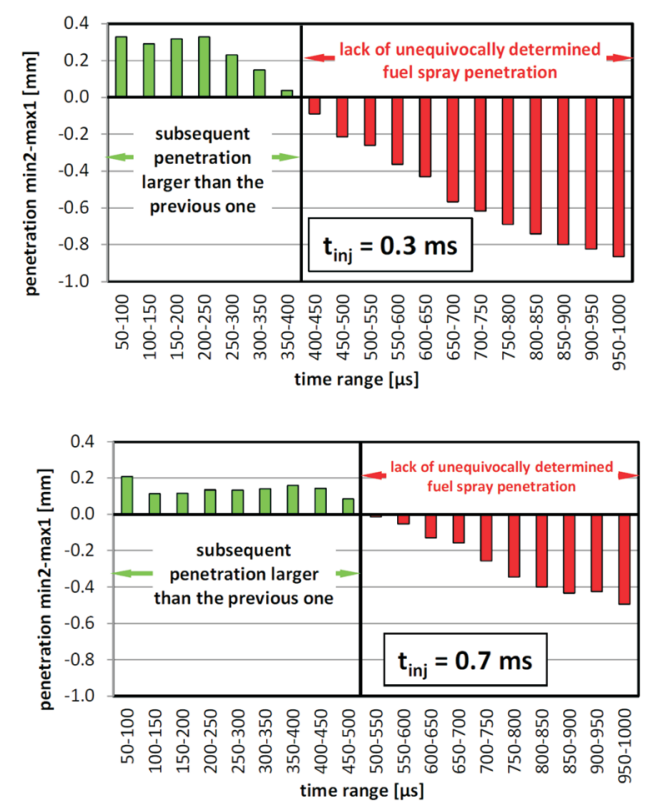

Fig. 9. The analysis of the possibility of determining the fuel spray penetration on the basis of optical analysis

\subsection{The assessment of average values of fuel spray velocity}

The velocity of fuel flowing out in proximity of the injector needle ranges from about $150 \mathrm{~m} / \mathrm{s}$ (on the basis of own tests [19, 20]) to $200 \mathrm{~m} / \mathrm{s}$ [8] depending on the pressure of the fuel injected and the back-pressure of the medium. The velocity of the air movement causing swirling of the atomized fuel spray (the end of its cone) is approximately $5-10 \mathrm{~m} / \mathrm{s}$.

In static tests (without mixture movement) with the use of a constant volume chamber the velocity tests were conducted taking into consideration the specified time after the start of the fuel injection. The test results were correlated with the fuel spray penetration as the spray velocity was determined on the basis of the difference of spray penetration in relation to the time of filming. The values contained in Fig. 10 show that the highest spray velocity is obtained after the fuel starts to flow from the atomizer. The value of the velocity obtained ranges from 45 to $75 \mathrm{~m} / \mathrm{s}$. With the propagation of fuel spray this velocity decreases. What is also characteristic is that the non-uniformity of this velocity decreases rapidly. After $0.1 \mathrm{~ms}$ from the start of the injection the spray velocity is variable within a minor range. The analysis shown in Fig. 11 indicates existence of significant non-uniformity of velocity $\mathrm{n}$ the initial phase of fuel injection. For the time up to $0.7 \mathrm{~ms}$ the values of the non-uniformity oscillate within the range of about $5 \%$. Although the fuel spray velocity decreases, the 
non-uniformity of the velocity in the final measuring range increases. This is caused by deeper radial penetration of fuel and reduced possibilities of determining the spray penetration (and the spray velocity).
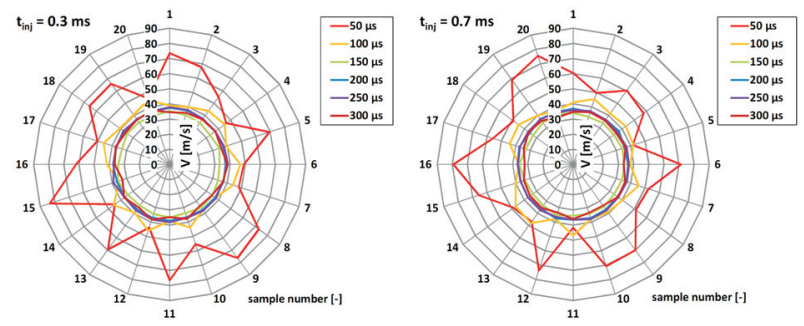

Fig. 10. The variation of fuel spray velocity at two injection durations for 20 consecutive repetitions
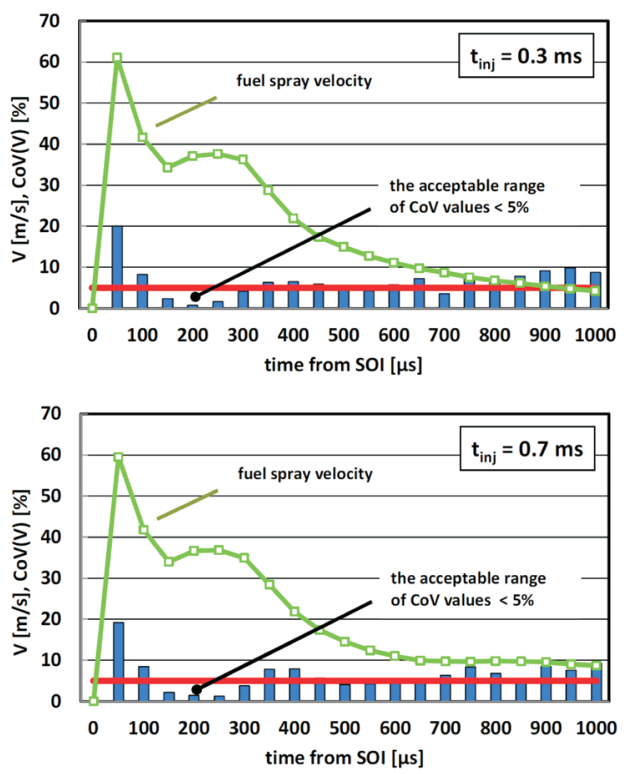

Fig. 11. The analysis of the fuel spray velocity and its non-uniformity at two amounts of injected fuel $(\mathrm{t}=0.3 \mathrm{~ms}$ and $\mathrm{t}=0.7 \mathrm{~ms})$

As the presented tests on fuel spray penetration show, the most significant non-uniformities occur during the start of the fuel injection. Therefore, in the further part of this paper is presented the detailed analysis of the phase of fuel flow from the injector.

\section{The assessment of the conditions at the start of the fuel injection}

The assessment was carried out during the injection, at $\mathrm{t}=0.05 \mathrm{~ms}$ after the injection start. The fuel spray penetration was analyzed for 20 consecutive doses of fuel injected. The results of this analysis are shown in Fig. 12. It shows that at the time of injection $\mathrm{t}=0.3 \mathrm{~ms}$ the maximum variation between the penetrations obtained can range from $2.2 \mathrm{~mm}$ to $4.1 \mathrm{~mm}$. For the increased dose of injected fuel the initial average spray penetration after time $t=0.05 \mathrm{~ms}$ is also $3 \mathrm{~mm}$.

The detailed radial analysis of such penetration is presented in Fig. 13. It shows quite considerable variation in the spray penetration even during one fuel injection. Taking into consideration such significant variations in spray penetration, it is impossible to place the injector and the spark plug in too close a proximity. There is no certainty if the fuel in such a short time reaches a specific point in space in order to ensure the ignitability of the mixture.
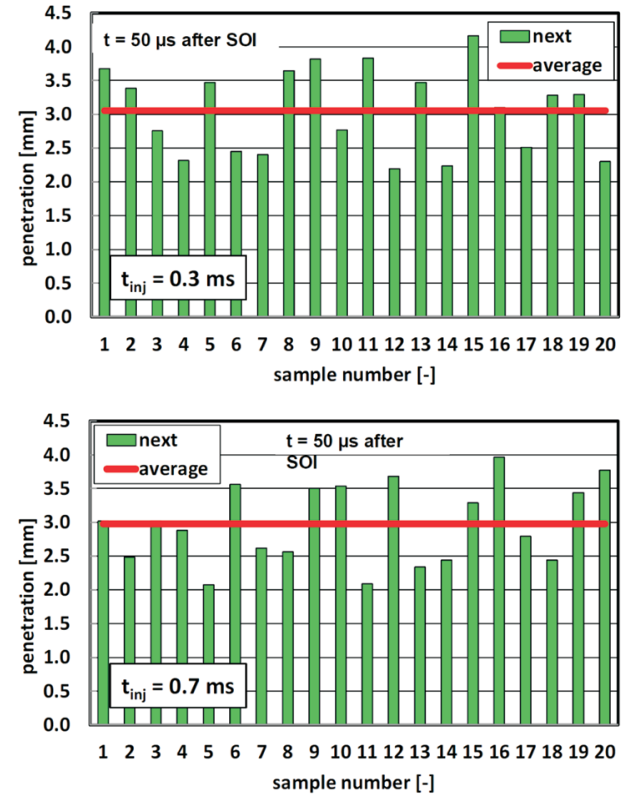

Fig. 12. The instantaneous and average value of the fuel spray penetration during injection for 20 subsequent measurements

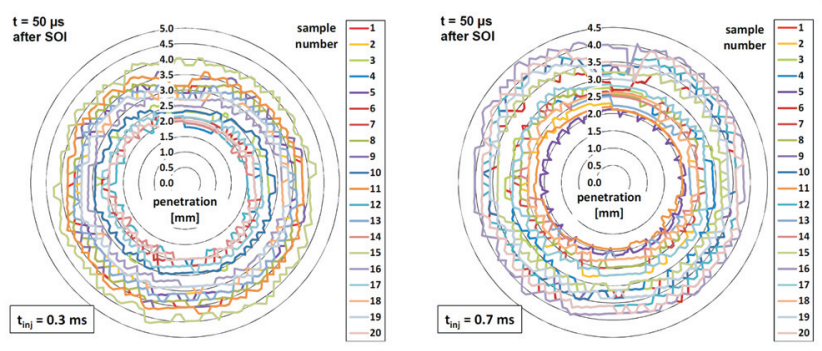

Fig. 13. The analysis of the radial fuel spray penetration after time $\mathrm{t}=0.05 \mathrm{~ms}$ at two fuel injection durations $(\mathrm{t}=0.3 \mathrm{~ms}$ and $0.7 \mathrm{~ms})$

\section{Summary}

The presented analysis of the non-uniformity of fuel spray penetration and non-uniformity of its velocity makes it possible to draw several conclusions:

1. The fuel injection from injectors of outward-opening type is characterized by a high repeatability of the radial spray penetration (deviation from the circular profile) for the time range from $t=0.1$ to $0.5 \mathrm{~ms}$ during injection of small doses, and for the time range from $\mathrm{t}=0.1$ to $0.2 \mathrm{~ms}$ for large doses of fuel injected (based on Fig. 5). This means that during the injection of small doses, it is necessary to shorten the time between the start of injection and the ignition. Injection of large doses at fuel pressure to air pressure ratio of 10:1 increases this non-uniformity (the critical value of which is estimated at $5 \%$ ).

2. Assessment of the average fuel spray penetration (based on Fig. 8) indicates the existence of a time, after which the value of non-uniformity of spray penetration falls below 
$5 \%$. This time, irrespective of the injected fuel amount, was determined at approximately $0.2 \mathrm{~ms}$.

3. The average value of the radial fuel spray penetration from the beginning of fuel injection is identified within the range of up to $0.4 \mathrm{~ms}$ (based on Fig. 9). This means that for the back-pressure the radial spray penetration is unequivocally increased up to the time pointed out above. In the subsequent time intervals it is impossible to determine unequivocally the spray penetration, which might be caused by high rate of petrol fuel evaporation as well as by the occurrence of swirling resulting from the air cone inside the atomized liquid.

4. Due to the high initial velocity of fuel spray (for time up to $\mathrm{t}=0.05 \mathrm{~ms}$ ) and the lack of a clear determination of its velocity it is not possible to obtain high efficiency of ignition in so short a time after the commencement of the injection. Significant deviations in determining the fuel spray velocity also ensues from significant non-uniformity of the spray penetration obtained after such time. It can also be caused by variations at the initiation of fuel flow from the injector. This may involve the need to increase the speed of recording in order to determine the exact beginning of fuel flow from the injector.

\section{Acknowledgements}

The author wishes to thank Prof. K. Wisłocki and MEng. P. Borowski, MEng. W. Cieslik, MEng. M. Skowron, MEng W. Bueschke from Poznań University of Technology for their participation in the research and critical, yet invaluable remarks on the analysis of optical investigations into fuel injection.

\section{Bibliography}

[1] Achleitner E., Bäcker H., Funaioli A. Direct injection systems for Otto engines. SAE Technical Paper 2007-01-1416, 2007, doi:10.4271/2007-01-1416.

[2] Aleiferis P.G., Serras-Pereira J., van Romunde Z., Caine J., Wirth M. Mechanisms of spray formation and combustion from a multi-hole injector with E85 and gasoline. Combustion and Flame. 2010, 157, 735-756.

[3] Arcoumanis C., Kamimoto T. Flow and combustion in reciprocating engines. Springer, Berlin - Heidelberg, Germany, 2008.

[4] Ashgriz N. Handbook of atomization and sprays. Theory and applications. Springer, New York 2011.

[5] Chen H., Reuss D.L., Sick V. Analysis of misfires in a direct injection engine using proper orthogonal decomposition. Experiments in Fluids. 2011, 51. doi:10.1007/s00348-0111133-z.

[6] El-Asrag H.A., Iannetti A.C., Apte S.V. Large eddy simulations for radiation-spray coupling for a lean direct injector combustor. Combustion and Flame. 2014, 161, 510-524.

[7] Gavaises M., Tonini S., Marchi A., Theodorakakos A., Bouris D., Matteucci L. Modelling of internal and near-nozzle flow of a pintle-type outwards-opening gasoline piezo-injector. International Journal of Engine Research. 2006, 7, doi:10.1243/14680874JER00306.

[8] Kim S., Kim Y., Lee J. Analysis of the in-cylinder flow, mixture formation and combustion processes in a spray-guided GDI engine. SAE Technical Paper 2008-01-0142, 2008, doi:10.4271/2008-01-0142.
[9] Marchi A., Nouri J., Yan Y., Arcoumanis C. Spray stability of outwards opening pintle injectors for stratified direct injection spark ignition engine operation. International Journal of Engine Research. 2010, 11. doi:10.1243/14680874JER605.

[10] Oh H., Bae C. Effects of the injection timing on spray and combustion characteristics in a spray-guided DISI engine under lean-stratified operation. Fuel. 2013, 107, 225-235. doi:10.1016/j.fuel.2013.01.019.

[11] Peterson B., Reuss D.L., Sick V. On the ignition and flame development in a spray-guided direct-injection spark-ignition engine. Combustion and Flame. 2014, 161, 240-255. doi:10.1016/j.combustflame.2013.08.019.

[12] Pielecha I. Modelling of gasoline fuel spray penetration in SIDI engines. International Journal of Automotive Technology. 2014, 15(1), 47-55. doi:10.1007/s12239-014-0005-y.

[13] Rotondi R., Hélie J., Leger C., Mojtabi M., Wigley G. Multihole gasoline direct injection spray plumes. 23rd Annual Conference on Liquid Atomization and Spray Systems, Brno, Czech Republic, 2010.

[14] Sauter W., Pfeil J., Velji A., Spicher U., Laudenbach N., Altenschmidt F., Schaupp U. Application of particle image velocimetry for investigation of spray characteristics of an outward opening nozzle for gasoline direct injection. SAE Technical Paper 2006-01-3377, 2006. doi:10.4271/2006-013377.

[15] Skogsberg M., Dahlander P., Denbratt I. Spray shape and atomization quality of an outward-opening piezo gasoline DI injector. SAE Technical Paper 2007-01-1409, 2007. doi:10.4271/2007-01-1409.

[16] VanDerWege B., Han Z., Iyer C., Muñoz R., Yi J. Development and analysis of a spray-guided DISI combustion system concept. SAE Technical Paper 2003-01-3105, 2003. doi: 10.4271/2003-01-3105.

[17] Waltner A., Lückert P., Schaupp U., Rau E., Kemmler R., Weller R. Future technology of the spark-ignition engine: spray-guided direct injection with piezo injector. The potential of synthetic fuels to meet future emission regulations, $27 \mathrm{st}$ International Vienna Engine Symposium 2006, Wien, Austria, 2006.

[18] Warnecke V., Achleitner E., Bäcker H. Development status of the Siemens VDO piezo injection system for spray-guided combustion. 27th International Vienna Motor Symposium, Wien Austria, 2006.

[19] Wisłocki K., Pielecha I., Czajka J., Maslennikov D. The influence of fuel injection parameters on the indexes of fuel atomization quality for a high pressure injection. SAE Technical Paper 2010-01-1499, 2010. doi:10.4271/2010-01-1499.

[20] Wisłocki K., Pielecha I., Czajka J., Maslennikov D. The qualitative spray characteristics of high-pressure gasoline injection system. 23rd European Conference on Liquid Atomization and Spray Systems - ILASS - Europe 2010, Brno 2010.

Ireneusz Pielecha, DSc., DEng. - professor at the Faculty of Machines and Transport of Poznan University of Technology.

e-mail: ireneusz.pielecha@put.poznan.pl 